ORIGINAL

\title{
Síndrome metabólico en personal de enfermería
}

\author{
María Escasany, María José Tumminello y Graciela Aída González* \\ División Alimentación y Dietoterapia. Hospital J uan A. Fernández, Buenos Aires, Argentina
}

Recibido el 11 de noviembre de 2011; aceptado el 9 de mayo de 2012

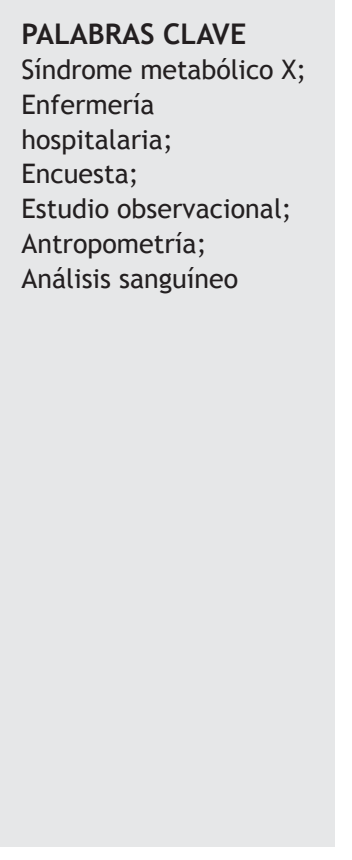

\begin{abstract}
Resumen
Objetivos: Estimar la prevalencia de síndrome metabólico (SM) entre las enfermeras del Hospital Juan A. Fernández (HJAF) y determinar si trabajo, descanso, dieta y estado de salud son sus predictores.

Material y métodos: Para el primer objetivo, el diseño del estudio es descriptivo, observacional y transversal. Para el segundo, comparación de muestras independientes, multivariable, observacional y transversal. Se estudió a 192 enfermeras entre octubre de 2008 y marzo de 2009. Se les realizó una encuesta autoaplicable con indicadores que podrían ser predictores de SM. Se tomaron mediciones antropométricas, presión arterial y extracción sanguínea para analizar glucemia en ayunas, colesterol unido a lipoproteínas de alta densidad y triglicéridos plasmáticos.

Resultados: El 35 y el $41 \%$ del personal de enfermería tenían sobrepeso y obesidad respectivamente. Un $92 \%$ del total tenía obesidad centroabdominal. La prevalencia de SM hallada es del 33,3\% (intervalo de confianza del 95\%, 26,7\%-40,5\%). Las afectas de SM tenían una media de edad de $53 \pm 9$ años. En el análisis bivariable se encontraron diferencias estadísticamente significativas entre el SM y las variables edad, tiempo de ejercicio profesional, tiempo trabajado en turno de noche y estudios realizados.

Conclusiones: La prevalencia de SM de enfermeras del HJAF fue de 64/192 (33,3\%; intervalo de confianza del $95 \%, 26,7 \%-40,5 \%$ ). Frente a la edad, los indicadores tiempo trabajado durante turno noche y estudios realizados no muestran diferencias estadísticamente significativas. Estos resultados indicarían que la edad es la variable más relevante para predecir la aparición de SM en la población de enfermeras.

(c) 2011 Asociación Española de Dietistas-Nutricionistas. Publicado por Elsevier España, S.L. Todos los derechos reservados.
\end{abstract}

\footnotetext{
*Autor para correspondencia.

Correo electrónico: licgonzalezg@gmail.com (G.A. González).
} 


\section{KEYWORDS}

Metabolic syndrome $X$, Nursing staff hospital, Questionnaire, Observational study, Anthropometry, Blood chemical analysis

\section{Metabolic Syndrome in Nurses}

\section{Abstract}

Objectives: To estimate the prevalence of metabolic syndrome (MS) in female nurses in the Hospital Juan A. Fernandez (HJAF), Buenos Aires, Argentina, and to determine whether work, rest, diet, and health, are predictive of it.

Materials and methods: For the first objective, a descriptive, observational and crosssectional study was conducted, and for the second, a multivariate cross-sectional observational multivariate analysis was made comparing independent samples. A total of 192 nurses were studied between October 2008 and March 2009. They completed a questionnaire that include indicators that could be predictors of MS. Anthropometric measurements, including blood pressure were taken, was well as a blood sample to analyze fasting glucose, $\mathrm{HDL}-\mathrm{C}$ and plasma triglycerides.

Results: It was found that $35 \%$ and $41 \%$ of nurses were overweight and obese, respectively. A total of $92 \%$ had centro-abdominal obesity. The prevalence of MS found was $33.3 \%$ $(95 \% \mathrm{Cl}, 26.7$ to 40.5$)$. Those who had this disease were between $53 \pm 9$ years. Statistically significant differences were found in the bivariate analysis between MS and the variables, age, length of service, time worked during night shift, and academic studies.

Conclusions: The prevalence of MS was 64/192 in HJAF nurses $(33.3 \%$ I 95\% Cl, 26.7-40.5). There were no statistically significant differences with the indicators of, age, "time worked during night shift", and "studies". These results suggest that age is the most important variable in predicting the onset of MS in the population of nurses.

(c) 2011 Asociación Española de Dietistas-Nutricionistas. Published by Elsevier España, S.L. All rights reserved.

\section{Introducción}

Considerando la alta prevalencia de sobrepeso y obesidad entre las enfermeras, se decidió realizar un estudio para estimar la prevalencia de síndrome metabólico (SM) en dicha población, ya que la conjunción de varias enfermedades o factores de riesgo en un mismo individuo aumenta la probabilidad de sufrir un evento cardiovascular o diabetes mellitus (DM).

Hay consenso general en que el SM se debe tratar preventivamente sin esperar a la aparición de DM y enfermedad cardiovascular. La modificación de los hábitos de vida tiende a normalizar todos los factores de riesgo, con lo que se retrasa la aparición de complicaciones irreversibles que llevan a un aumento en la morbilidad y la mortalidad general.

Es de suma importancia estimar la prevalencia de SM entre las enfermeras del Hospital J.A. Fernández (HJAF) para, en un futuro, realizar intervenciones de educación nutricional y acciones que favorezcan a un mejor estado de salud en dicha población.

Se denomina SM al conjunto de alteraciones metabólicas constituido por obesidad de distribución central, disminución de las concentraciones del colesterol unido a lipoproteínas de alta densidad (cHDL), elevación de las concentraciones de triglicéridos, aumento de la presión arterial (PA) e hiperglucemia ${ }^{1,2}$.

Desde la primera definición oficial de SM realizada por el Grupo de Trabajo de la Organización Mundial de la Salud (OMS) en $1999^{3}$, se han propuesto diversas definiciones alternativas. Esta situación llevó a la Federación Internacional de Diabetes (FID) a elaborar una nueva definición que pudiera tener alcance universal, expuesta durante el Primer Congreso Internacional de Prediabetes y SM celebrado en Berlín, Alemania, en abril de $2005^{4}$.

Según la FID, una persona padece SM si, además de obesidad centroabdominal, que es un requisito, presenta dos o más factores ${ }^{5,6}$.

La prevalencia de SM en la población adulta general de Estados Unidos es del 25\%; de igual manera, 1/5 españoles padece SM según el Presidente de la Sociedad Española de Aterosclerosis, Dr. Luis Massana, y $1 / 5$ argentinos también, según el Dr. Litwak, Jefe de la Sección Diabetes y Metabolismo del Hospital Italiano7,8.

Entre los factores determinantes de la aparición de enfermedades crónicas, la OMS ha reconocido los relacionados con el estado nutricional y la actividad física como algunos de los más importantes. En la mayoría de los casos, la expresión de SM ocurre en individuos obesos ${ }^{9}$.

La obesidad y, fundamentalmente, el exceso de grasa visceral predisponen a hipertensión arterial (HTA), DM2 y trastornos del colesterol, lo que favorece el desarrollo de enfermedad cardiovascular (ECV) aterosclerótica ${ }^{10}$. Considerando que la obesidad y el sedentarismo son factores de riesgo subyacentes en la ruta patogénica de este síndrome, la modificación de los hábitos de vida es una intervención de primera línea en la prevención y el tratamiento de la insulinorresistencia, la hiperglucemia, la dislipemia aterogénica y la HTA ${ }^{11}$.

Múltiples estudios han investigado el desarrollo de SM en profesionales del equipo de salud. ${ }^{12-13}$

Un estudio que evaluó su prevalencia y los factores de riesgo cardiovascular en 414 trabajadores de la salud (auxi- 
liares de enfermería, enfermeros y médicos del Hospital Universitario amón González de Valencia, Colombia) halló el síndrome en el 13,2\% de la población, diagnosticado según los criterios del NCEP-ATP III ${ }^{14}$.

Otro estudio realizado en México, en el cual se incluyeron 370 casos entre el personal de enfermería del Hospital Médica Sur (el 97,8\% mujeres), encontró que la prevalencia de SM era del $12,4 \%$ la de sobrepeso (índice de masa corporal $[I M C]>27)$, del 34,9\%, y la de obesidad (IMC > 30), del $20 \%{ }^{15}$. En el mismo país, se buscó conocer la prevalencia de SM entre los trabajadores de la salud de un hospital general de agudos de Celaya. El estudio incluyó a 200 trabajadores de la salud. La prevalencia general de SM según criterios del NCEP-ATP III fue del 29,5\% ${ }^{16}$.

Según la OMS, los profesionales de enfermería, como defensores de la salud de las personas, deben fomentar en su propia persona un adecuado autocuidado que promueva el bienestar biopsicosocial, para desarrollar una personalidad terapéutica que transmita e inspire salud ${ }^{17,18}$. El síndrome de burnout fue descrito por el psiquiatra estadounidense Herbert Freudenberger en 1974 y lo han estudiado con gran interés innumerables autores desde principios de la década de los ochenta ${ }^{19}$. Diferentes estudios han encontrado diferencias significativas al relacionar los componentes del burnout con el IMC ${ }^{20,21}$.

Un estudio realizado en la Universidad de oma comparó dos grupos de personal de enfermería en cuanto a sus riesgos de sufrir SM ( $n$ 202) enfermeros que trabajan durante el día frente a enfermeros que trabajan durante la noche. La investigación demostró que el trabajo durante la noche es un riesgo adicional de SM para los enfermeros (odds ratio $[\mathrm{OR}]=4,10$; intervalo de confianza del 95\% [IC95\%], 1,34$12,55 \mathrm{p} \quad 0,01)^{22}$

De acuerdo con informes previos, los programas de detección y tratamiento oportuno de los factores de riesgo modifican notablemente las posibilidades de que se desarrolle $\mathrm{SM}^{23}$. Una vez diagnosticado el SM, la alimentación acompañada de un estilo de vida sano que incluya actividad física regular puede revertir o reducir significativamente el riesgo cardiovascular que el síndrome implica para la salud $^{9}$. Teniendo en cuenta la prevalencia de sobrepeso y obesidad entre las enfermeras, se decidió realizar un estudio para estimar la prevalencia de SM y establecer diversos indicadores laborales, de descanso y alimentarios.

\section{Material y $\mathrm{m}$ todos}

Se estudió a la población formada por 192 mujeres del personal de enfermería del HJAF entre octubre de 2008 y marzo de 2009.

Criterios de inclusión personal de enfermería de sexo femenino (licenciadas en Enfermería, enfermeras profesionales o auxiliares de enfermería). Criterios de exclusión estar cursando un embarazo y no desear participar del estudio. Criterios de eliminación no realizar los estudios completos necesarios.

Se estudió a las enfermeras que desempeñan sus funciones en el HJAF en el periodo de octubre de 2008 a marzo de 2009. La muestra se obtuvo por muestreo consecutivo entre quienes cumplían con los criterios mencionados.
Dado que se realizó un análisis multivariable, se aplicó una fórmula de estimación para estimar el tamaño muestral ${ }^{24}$. Para el primer objetivo, el diseño de investigación fue descriptivo, observacional y transversal. Para el segundo, el diseño de investigación fue de comparación de muestras independientes, multivariable, observacional y transversal ${ }^{25}$.

Se entregó una encuesta autoaplicable, en la que se completaron datos personales y se especificó sobre la existencia de diagnóstico médico de DM y/o dislipemias y/o HTA o tratamiento farmacológico para control glucémico, lipídico o de la PA. En ella se hacían preguntas cerradas sobre diversos indicadores laborales, alimentarios, de descanso y de salud. Se dejó asentada la participación voluntaria en el estudio mediante la firma obligatoria del consentimiento informado.

Se realizaron las siguientes mediciones antropométricas peso corporal, talla y circunferencia de cintura (CC). El peso corporal se tomó utilizando una báscula de capacidad para $150 \mathrm{~kg}$ (CAM), con una exactitud de $100 \mathrm{~g}$. Se pesó a las participantes con la vestimenta de trabajo habitual (ambo), sin calzado ni abrigo. La talla se tomó con el estadiómetro de la báscula (precisión, $0,1 \mathrm{~cm}$ ). La medición de la CC se realizó en el punto medio entre el reborde costal y la cresta iliaca $(\mathrm{OMS})^{26}$. La PA se midió con un tensiómetro. Se tomó la precaución de que el paciente se encontrara relajado y que no hablara durante el procedimiento de medición ${ }^{25,27}$. Se solicitó una extracción de sangre tras $12 \mathrm{~h}$ de ayuno para evaluar colesterol total, colesterol unido a lipoproteínas de baja densidad (cLDL), cHDL, triglicéridos y glucemia en ayunas.

\section{ariables rele antes y o eracionali ación}

Variable dependiente: SM se consideró SM en caso de obesidad central más concurso de dos de los restantes factores, y ausencia de SM cuando no había obesidad central o la había junto con sólo uno o ninguno de los restantes factores (tabla 1).

Variables independientes (indicadores): laborales, de descanso, alimentarios, de salud.

Variable a controlar: edad en años, según lo informado por la participante.

Los datos fueron volcados a una base de datos (Microsoft Excel 97) y luego se analizaron empleando el paquete estadístico (SPSS 11.5, Medcalc 9.1 y VCCstat 2.0). Cuando fue necesario, se realizaron pruebas de significación (pruebas de la $t$ de Student y de la $\chi^{2}$ ). Posteriormente se realizó análisis de regresión logística multivariable para las variables correspondientes con $p<0,05$, definida como variable dependiente la presencia de SM.

\section{Resultados}

El personal de enfermería del HJAF está constituido por 345 mujeres ( $80 \%$ ) y 86 varones (20\%). Del total de enfermeras, 214 completaron la encuesta (el $62,03 \%$ del total). La muestra final quedó constituida por 192 enfermeras (el $55,65 \%$ del total).

Se estimó la prevalencia de SM entre las encuestadas, que fue $64 / 192$ (33,3\%; IC95\%, 26,7\%-40,5\%) (fig. 1).

El promedio de edad de las enfermeras que no tenían SM era 4411 años, mientras que entre las que sí lo tenían era 
abla Definición mundial de síndrome metabólico propuesta por la FID ${ }^{4}$

\begin{tabular}{|c|c|}
\hline $\begin{array}{l}\text { 1. Obesidad central } \\
\text { Circunferencia de la } \\
\text { cinturaa: con especificidad } \\
\text { respecto a los distintos } \\
\text { grupos étnicos }\end{array}$ & $\begin{array}{l}\geq 80 \mathrm{~cm} \text { (mujeres) } y \geq 90 \mathrm{~cm} \\
\text { (varones) }\end{array}$ \\
\hline $\begin{array}{l}\text { Más dos de los siguientes } \\
\text { factores }\end{array}$ & \\
\hline $\begin{array}{l}\text { 2. Aumento de los } \\
\text { triglicéridos }\end{array}$ & $\begin{array}{l}\geq 150 \mathrm{mg} / \mathrm{dl} \text { o tratamiento } \\
\text { específico de esta alteración } \\
\text { lipídica }\end{array}$ \\
\hline 3. Disminución del cHDL & $\begin{array}{l}<50 \mathrm{mg} / \mathrm{dl} \text { (mujeres) o } \\
<40 \mathrm{mg} / \mathrm{dl} \text { (varones) o } \\
\text { tratamiento específico de } \\
\text { esta alteración lipídica }\end{array}$ \\
\hline $\begin{array}{l}\text { 4. Aumento de la presión } \\
\text { arterial }\end{array}$ & $\begin{array}{l}\text { Sistólica } \geq 130 \mathrm{mmHg} ; \\
\text { diastólica } \geq 85 \mathrm{mmHg} \text { o } \\
\text { tratamiento de hipertensión } \\
\text { diagnosticada previamente }\end{array}$ \\
\hline $\begin{array}{l}\text { 5. Incremento de la } \\
\text { glucemia }\end{array}$ & $\begin{array}{l}\text { Glucemia en ayunas } \\
\geq 100 \mathrm{mg} / \mathrm{dl}^{\mathrm{b}} \text { o DM2 } \\
\text { diagnosticada previamente }\end{array}$ \\
\hline
\end{tabular}

DM diabetes mellitus FID Federación Internacional de Diabetes IMC índice de masa corporal SM síndrome metabólico.

a Si el IMC es 30, se puede presumir la obesidad central y no es necesario medir la circunferencia de la cintura.

bSi la glucemia en ayunas es $>100 \mathrm{mg} / \mathrm{dl}$, se recomienda la realización de una prueba de tolerancia a la glucosa oral, aunque no es necesaria para definir la presencia del síndrome. En la práctica clínica también es aceptable la demostración de la alteración de la tolerancia a la glucosa, pero en todos los estudios epidemiológicos relativos a la prevalencia de SM se debe utilizar únicamente la glucemia en ayunas y/o la presencia de DM previamente diagnosticada para evaluar este criterio.

$53 \pm 9$ años (fig. 2). La diferencia entre los promedios de edades fue significativamente superior entre las que sufrían el síndrome.

Para establecer si los indicadores laborales, de descanso, alimentarios y de salud son predictores de SM, se realizó un análisis bivariable para determinar si existía asociación con la enfermedad.

En la figura 3 se puede observar el análisis de la relación entre los estudios realizados (tipo de título) y el SM. Se encontraron diferencias estadísticamente significativas, con menor presencia de SM entre el personal de enfermería con mayor instrucción.

El tiempo de ejercicio profesional se asoció de manera estadísticamente significativa con la presencia de SM, y el tiempo de ejercicio de la profesión entre las enfermeras con SM resultó ser superior. El promedio de años de trabajo ejerciendo sus actividades en turno de noche fue significativamente superior entre las enfermeras con SM que entre aquellas sin él.

La frecuencia semanal de consumo de comidas compradas en kioscos, bares o rotiserías no tuvo relación con la

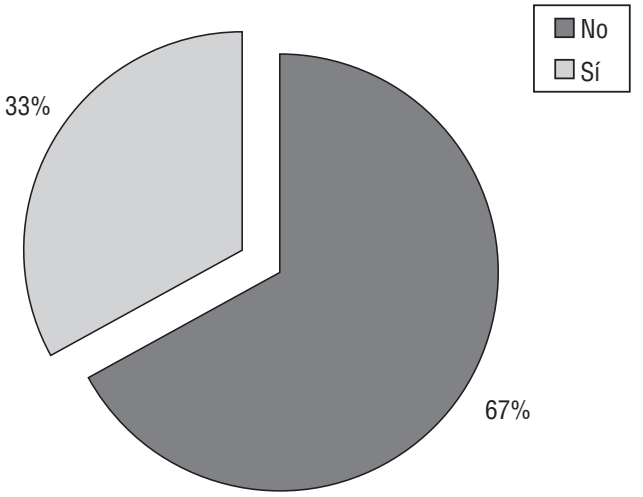

i ura Distribución de la población según prevalencia de síndrome metabólico (n 192).

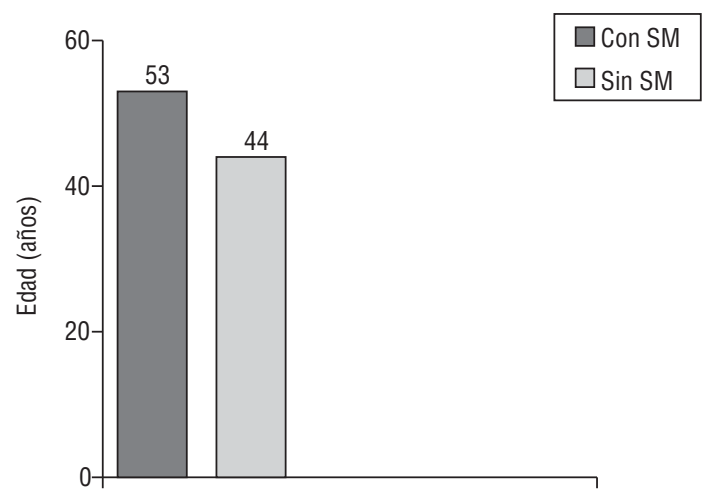

i ura Distribución de la población según promedio de edad y prevalencia de síndrome metabólico (SM) (n 192).

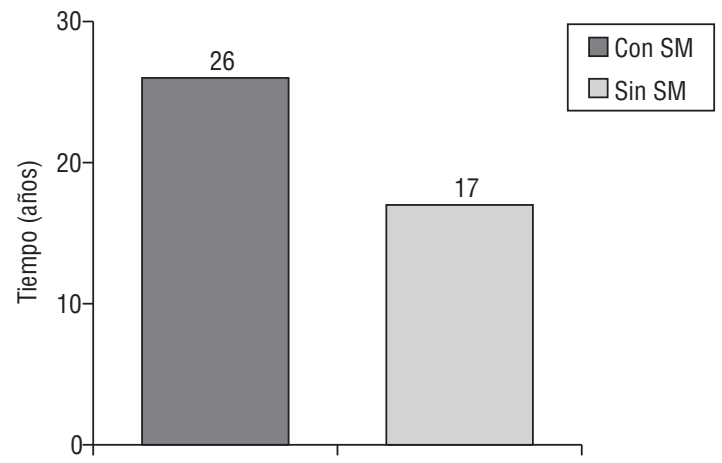

i ura Promedio de años de ejercicio profesional en relación con el síndrome metabólico (SM) (n 192).

presencia del SM. El hecho de comer por aburrimiento o ansiedad o para mantenerse despierto durante las horas de trabajo no tuvo relación con la presencia del síndrome.

La percepción de la influencia positiva en relación al trabajo de enfermería en las personas evaluadas no tuvo relación con la presencia de SM. 
La colinealidad de la variable años de ejercicio de la profesión con el resto de las variables indicaría que la edad es la variable más relevante para predecir la aparición de SM en la población de enfermeras.

\section{Discusión}

La motivación principal para realizar el presente estudio fue la observación directa de la gran prevalencia de sobrepeso y obesidad entre las mujeres del personal de enfermería del HJAF. Lo mismo llevó a la reflexión sobre la probabilidad de encontrar en esta población un riesgo de SM adicional. Las mediciones antropométricas realizadas confirmaron lo observado, ya que el $76 \%$ del personal de enfermería que participó en el estudio tuvo diagnóstico nutricional de sobrepeso u obesidad (el $35 \%$ en sobrepeso y el $41 \%$ en obesidad franca).

La prevalencia de SM de la población estudiada fue del $33,3 \%$ (IC95\%, 26,7\%-40,5\%), es decir, un riesgo equivalente a que $4 / 10$ enfermeras contraigan SM.

La revisión bibliográfica realizada para este estudio indica que $1 / 5$ argentinos padece el síndrome. Por lo tanto, la prevalencia de SM hallada entre las mujeres del personal de enfermería demuestra que el riesgo de padecerlo es 2 veces mayor que para la población general.

Los hallazgos revelan una asociación estadísticamente significativa de ciertos indicadores al analizarlos independientemente con el SM (análisis bivariable) la edad de las enfermeras, el tiempo de ejercicio profesional (en años), el tiempo trabajado en turno de noche (en años) y los estudios realizados (tipo de título).

En cuanto al tipo de título, a mayor grado de instrucción se podría esperar mayores cuidados higiénico-dietéticos y de salud para con la propia persona.

Los otros dos indicadores con asociación estadísticamente significativa en relación con el SM en el análisis bivariable, tiempo trabajado en turno de noche (en años) y los estudios realizados (tipo de título), ingresaron al modelo multivariable por no presentar colinealidad. Sin embargo, ambos perdieron significación estadística frente a la edad de las enfermeras.

Es importante haber detectado conductas perjudiciales como el consumo de panificados con grasas saturadas y exceso de sal, entre otras, y conocer que la mayoría de las enfermeras no consume hortalizas en cantidad suficiente.

Si entendemos que la edad (con la significación biológica que el paso de los años tiene en el organismo) no puede modificarse, se considera particularmente importante lograr modificar la suma de "factores crónicos" durante todos los años de ejercicio profesional, independientemente de la edad. La meta sería mejorar el estado de salud de las enfermeras y prevenir "hábitos alimentarios crónicos perjudiciales en las enfermeras más jóvenes.

Se considera necesario continuar estudiando en este plano todas las posibles asociaciones de conductas y prácticas que incidan en una mayor prevalencia poblacional de SM.

\section{Conflicto de intereses}

Los autores declaran no tener ningún conflicto de intereses.

\section{iblio ra a}

1. Eckel R, et al. The metabolic syndrome. Lancet. 2005;365:141528.

2. immet $P$, et al. Mainstreaming the metabolic syndrome a definitive definition. Med J Aust. 2005;183:175-6.

3. Definition, diagnosis and classification of diabetes mellitus and its complications. eport of a $\mathrm{HO}$ consultation. Geneva orld Health Organization 1999.

4. Definición mundial de consenso para el síndrome metabólico. ev Panam Salud Publica. 200518.

5. The IDF consensus worldwide definition of the metabolic syndrome actualización Mar 2009 . International Diabetes Federation 2005. Disponible en www.idf.org

6. Alberti K, et al. The metabolic syndrome: a new world-wide definition from the International Diabetes Federation consensus. Lancet. 2005;366:1059-62.

7. Latorraga J. Una mirada del síndrome metabólico desde la nutrición y el paciente. Tres Arroyos 2004.

8. Machado JE, Moncada JC, Mesa G. Patrones de prescripción de antilipémicos en un grupo de pacientes colombianos. ev $\mathrm{Pa}$ nam Salud Pública. 200823.

9. García-García E, et al. La obesidad y el síndrome metabólico como problema de salud pública, una reflexión. Salud Pública de México. 200850.

10. Pisabarro, et al. Primera encuesta nacional de sobrepeso y obesidad. Rev Med Uruguay. 2000;16:31-8.

11. Matía Martín P, et al. Nutrición y síndrome metabólico. ev Esp Salud Publica. 2007.

12. www.fmed.uba.ar actualizada, Mar 2009.

13. www.hospitalitaliano.org.ar actualizada, Mar 2009.

14. incón Peña 0 , et al. Valoración del riesgo cardiovascular global y prevalencia de síndrome metabólico en trabajadores de la salud del Hospital Universitario amón González Valencia. Acta Médica Colombiana. 200429 312-21.

15. Méndez Sánchez N, et al. High prevalence of overweight, obesity, and biochemical risk factors for cardiovascular disease among young nurses importance of metabolic syndrome. Med Sur. 200815 185-90.

16. Padierna-Luna JL, et al. Prevalence of metabolic syndrome in health employees. Rev Med Inst Mex Seg Soc. 2007;45:593-9.

17. Educación Sanitaria Popular. Ginebra: Organización Mundial de la Salud; 1955. p. 364-71.

18. León omán C. Cuidarse para no morir cuidando. ev Cuba Enferm. 2007;23.

19. Arias Galicia F, et al. Un estudio de agotamiento (burnout) profesional en el personal de salud en el estado de Morelos. Universidad Autónoma del Estado de Morelos; 2006.

20. Fong $E$, et al. Prevalencia de sobrepeso y obesidad en el personal de enfermería. Med Int Mex. 2006;22:81-4.

21. Vela Bueno A, et al. Implicaciones biológicas y psicosociales del trabajo a turnos en la mujer Un estudio en enfermeras. Fundación General de la Universidad Autónoma de Madrid, 20052007.

22. La Sala M, et al. Metabolic syndrome and work: identification of populations at risk. Roma. 2007;29:445-7.

23. Fanghanel-Salmon $\mathrm{G}$, et al. Prevalencia de factores de riesgo de enfermedad coronaria en trabajadores del Hospital General de México. Salud Pública Méx. 1997;39.

24. Dawson-Saunders B, et al. Bioestadística médica. México El Manual Moderno 2002.

25. Castiglia, Principios de Investigación Biomédica. 2. ${ }^{\text {a ed. }} 1998$.

26. Girolami D. Fundamentos de valoración nutricional y composición corporal. Buenos Aires El Ateneo 2003.

27. Bazzino O, et al. Síndrome metabólico. Biomedicina. 2006; 2 214-21. 